\title{
Astrochemistry of dimethyl ether
}

\author{
Z. Peeters ${ }^{1}$, S. D. Rodgers ${ }^{2}$, S. B. Charnley ${ }^{2}$, L. Schriver-Mazzuoli ${ }^{3}$, A. Schriver ${ }^{3}$, J. V. Keane ${ }^{2}$, and P. Ehrenfreund ${ }^{1}$ \\ 1 Leiden Institute of Chemistry, PO Box 9502, 2300 RA, Leiden, The Netherlands \\ e-mail: peeters@strw.leidenuniv.nl \\ 2 Space Science and Astrobiology Division, NASA Ames Research Center, MS 245-3, Moffett Field, CA 94035, USA \\ 3 Université P. et M. Curie, Department of Physical Chemistry, Case 22, 4 place Jussieu, 75005 Paris, France
}

Received 17 June 2005 / Accepted 31 August 2005

\section{ABSTRACT}

Dimethyl ether (DME, $\mathrm{CH}_{3} \mathrm{OCH}_{3}$ ) is one of the largest organic molecules detected in the interstellar gas and shows high abundances in starforming regions, known as hot molecular cores. The observed DME might be formed on grains or by secondary gas phase reactions from a precursor molecule, which in turn was sublimed into the gas phase from the grain surface. Studies on the stability and degradation pathways of DME therefore provide important constraints on the evolutionary cycle of large organic molecules and chemical pathways in the interstellar medium. We studied the UV photodestruction rate of DME in a solid argon matrix. DME was destroyed with a half-life of 54 seconds under laboratory conditions, which corresponds to $8.3 \times 10^{5}$ years in a dense cloud. We discuss the UV photochemistry of DME in the context of two issues: its formation mechanism and its chemistry in hot cores. Chemical models of shielded hot core regions indicate that UV photodestruction is relatively unimportant for DME, even by the internally-generated radiation field. These models clearly show that gas phase processes are almost certainly responsible for the formation of interstellar DME.

Key words. astrochemistry - methods: laboratory - ISM: molecules

\section{Introduction}

In the energetic environments of forming massive and lowmass protostars, so-called hot molecular cores, thermal heating or sputtering in shock waves can liberate the molecular ice mantles that cover interstellar dust grains (e.g. van Dishoeck \& Blake 1998; Ehrenfreund \& Charnley 2000). Observations of these regions can therefore be used to probe the nature of grainsurface chemistry. The desorbed molecules can initiate distinctive gas phase reaction pathways, that can lead to the growth of highly complex organic molecules (Charnley et al. 1995), oxygen-nitrogen molecular differentiation (Charnley et al. 1992; Caselli et al. 1993), and the possibility of comparing dynamical time-scales through "chemical clocks" (Charnley 1997b; Hatchell et al. 1998).

Dimethyl ether $\left(\mathrm{DME}, \mathrm{CH}_{3} \mathrm{OCH}_{3}\right)$ is ubiquitous in regions of massive star formation where it is found to exist solely in hot cores (e.g. Turner 1991; Sutton et al. 1995; Nummelin et al. 2000). It has also been detected recently in the hot core (viz. the "hot corino") of the low-mass binary system IRAS 16293-2422 (Cazaux et al. 2003; Kuan et al. 2004). Observations of comet Hale-Bopp have failed to detect DME in the coma (Crovisier et al. 2004) and it is also undetected in cold molecular clouds (e.g. Friberg et al. 1988).

We are currently undertaking a study on the photostability of complex molecules, to assess their survivability in different astronomical environments and delineate possible formation mechanisms (Ehrenfreund et al. 2001a,b; Peeters et al. $2003,2005)$. As a part of this program, we have now extended our previous experimental work to consider the photochemistry of DME (Schriver-Mazzuoli et al. 2002; Schriver et al. 2004). Infra-red (IR) spectroscopic studies of DME have been performed previously in the laboratory in argon and ice matrices (Schriver-Mazzuoli et al. 2002; Schriver et al. 2004). In those experiments we determined IR spectroscopic features and photoproducts upon irradiation with UV. In this paper we will put those measurements into astrophysical context using quantitative measurements concerning the UV stability of DME in the gas phase.

In the following sections we will describe the experiments and results of UV photolysis experiments of DME in a solid argon matrix. As a test of the putative grain-surface production of DME in star-forming regions, IR spectra of DME/ $\mathrm{H}_{2} \mathrm{O}$ mixtures will be compared to recent spectra of those regions. Finally, the astronomical application of laboratory and theoretical studies to the role of DME chemistry in hot cores will be reconsidered.

\subsection{The origin of DME}

DME was proposed by Blake et al. (1987) to originate in the gas phase. Methyl cation transfer to methanol and 
formaldehyde leads to DME and methyl formate $\left(\mathrm{HCOOCH}_{3}\right)$, respectively, after electron dissociative recombinations. The reaction

$$
\mathrm{CH}_{3} \mathrm{OH}_{2}^{+}+\mathrm{CH}_{3} \mathrm{OH} \longrightarrow \mathrm{CH}_{3} \mathrm{OCH}_{4}^{+}+\mathrm{H}_{2} \mathrm{O}
$$

is well-studied in the laboratory with a measured rate coefficient (Karpas \& Mautner 1989). Its inclusion in chemical models where methanol molecules are evaporated from grain surfaces can explain the high abundances of DME and methyl formate $\left(\sim 10^{-8}-10^{-7}\right)$, as well as the $\mathrm{CH}_{3} \mathrm{OCH}_{3} / \mathrm{CH}_{3} \mathrm{OH}$ abundance ratios of $\sim 0.02-0.1 \%$, that are typically observed (Charnley et al. 1992; Caselli et al. 1993).

However, observations of the $\mathrm{G} 34.3$ and $\mathrm{W} 3\left(\mathrm{H}_{2} \mathrm{O}\right)$ cores appear to indicate that their methanol abundances are lower than in other cores and comparable with those of its putative daughter molecules (MacDonald et al. 1996; Helmich \& van Dishoeck 1997). As models predict that at most a few per cent of the evaporated methanol is converted to DME and methyl formate (Charnley et al. 1992), it would be difficult to reproduce the DME abundance in G34.3 even with the higher methanol abundance determined by Mehringer \& Snyder (1996). There are also problems in interpreting the DME and methyl formate abundances of low-mass "hot corinos" within the standard picture. Observations of $\mathrm{HCOOCH}_{3}$ and $\mathrm{CH}_{3} \mathrm{OCH}_{3}$ in IRAS 16293-2422 led Cazaux et al. (2003) to derive $\mathrm{CH}_{3} \mathrm{OCH}_{3} / \mathrm{CH}_{3} \mathrm{OH}$ and $\mathrm{HCOOCH}_{3} / \mathrm{CH}_{3} \mathrm{OH}$ ratios close to unity. Cazaux et al. (2003) concluded that the IRAS 16293-2422 core is not old enough for post-evaporation gas phase chemistry to have formed these molecules and hence that, like methanol, they are the products of grain-surface reactions.

The proposed gas phase synthesis of $\mathrm{HCOOCH}_{3}$ is unlikely (Charnley 1997a), since experiments demonstrate that methyl cation transfer to $\mathrm{H}_{2} \mathrm{CO}$ does not occur (Karpas \& Mautner 1989, see also Horn et al. 2004). An alternative pathway to $\mathrm{HCOOCH}_{3}$ has been suggested (Charnley 1997a), involving the abundant mantle molecule $\mathrm{HCOOH}$ (Schutte et al. 1996) instead of $\mathrm{H}_{2} \mathrm{CO}$.

$$
\mathrm{CH}_{3} \mathrm{OH}_{2}^{+}+\mathrm{HCOOH} \longrightarrow \mathrm{CH}_{3} \mathrm{OCHOH}^{+}+\mathrm{H}_{2} \mathrm{O} \text {. }
$$

A surface origin for $\mathrm{HCOOCH}_{3}$ is possible since this molecule is observed in comets (e.g. Bockelée-Morvan et al. 2005) and coma chemistry calculations show that it must emanate from the nuclear ices (Rodgers \& Charnley 2001b). An important question is therefore whether there is sufficient time, postevaporation, for hot core chemistry to synthesise the observed abundances of $\mathrm{HCOOCH}_{3}$, or whether it too must be formed on grains?

The build-up of a molecule as complex as DME is unlikely to proceed efficiently on cold grain surfaces by addition reactions beginning from single atoms (e.g. Charnley 1997a). Hydrogen atom additions to an unsaturated precursor molecule, as occurs for example in the formation of methanol in $\mathrm{CO}$ ices (Watanabe \& Kouchi 2002), is also debatable. The known candidates containing a C-O-C structure (Ikeda et al. 2001) are more likely to lead to other stable products before six hydrogens are present, for example the reduction of ethylene oxide to oxirane (Charnley 2001). As any alternative precursors would require major structural rearrangements, DME may be assembled on grains by the association of simple radical functional groups, for example between methoxy and methyl radicals (e.g. Allen \& Robinson 1977; Sorrell 2001; Hollis \& Churchwell 2001). These radicals would have to be produced from abundant pre-existing molecules (i.e. methanol and methane) and so a source of energy is necessary, both to break chemical bonds and to provide mobility to the products. Irradiation of ices, either by heavy cosmic ray particles or by UV photons, has been studied extensively in the laboratory (e.g. Allamandola et al. 1997; Gerakines et al. 2001; Ehrenfreund et al. 2001b). In fact, experiments involving UV photolysis and warming of interstellar ice mixtures have shown that the IR signatures of methyl ethers can be produced (Bernstein et al. 1995). Although the identification of individual compounds has not been made, it is very likely that DME is amongst them. Thus, experiments show that photolysis and photostability could both play a role in the solid state production of DME. In this paper, we investigate this further based on our previous experimental work.

\subsection{DME in hot molecular cores}

Whether or not DME is formed solely on grains or in gas phase reactions can be important for understanding its chemical evolution in hot cores (and "hot corinos"). The utility of gaseous DME as a "chemical clock" for the post-evaporation phase lies in the fact that it is only formed from methanol, whose abundance in both the solid and gaseous phases can be measured. This means that the evolution of the $\mathrm{CH}_{3} \mathrm{OCH}_{3} / \mathrm{CH}_{3} \mathrm{OH}$ abundance ratio in hot cores depends mainly on the cosmic-ray ionization rate and the density, and less so on the temperature. Model calculations of DME formed in ion-molecule chemistry, typically suggest post-evaporation ages of a few times $10^{4}$ years for hot cores, based on the time-scale where the DME abundance peaks (Charnley et al. 1992).

However, if DME is formed solely on grains by some unknown process, its gas phase kinetics will be markedly different. In the gas phase, DME is destroyed primarily in reactions with $\mathrm{H}^{+}, \mathrm{He}^{+}$and $\mathrm{C}^{+}$and by protonation by $\mathrm{H}_{3}^{+}, \mathrm{HCO}^{+}, \mathrm{H}_{3} \mathrm{O}^{+}$ and $\mathrm{CH}_{3} \mathrm{OH}_{2}^{+}$. In electron recombinations, protonated DME can also recombine to methanol, leading to a net loss (Le Teuff et al. 2000). Neglect of DME gas phase formation through reaction 1 therefore leads to kinetics where the evaporated DME abundance suffers an exponential decline over the age of the core. Thus, in this case higher DME abundances can be expected to occur much earlier in the hot core lifetime.

Although hot molecular cores have high visual extinctions and thus effectively shield against the interstellar UV field, the photochemistry of gaseous DME can still become an issue in determining its evolution, irrespective of the actual formation mechanism. At the high densities of these regions $\left(\sim 10^{6}-10^{8} \mathrm{~cm}^{-3}\right)$, simple estimates indicate that photodestruction by the weak flux of Prasad-Tarafdar UV photons (Prasad \& Tarafdar 1983) can be competitive with destruction in the above ion-molecule reactions. Furthermore, the stellar UV radiation field will be important for the chemistry, either close to forming protostars (Stäuber et al. 2004), or near the end of the 
hot core phase when an ultracompact $\mathrm{H}$ II region starts to form (Kurtz et al. 2000).

\section{Experimental}

Matrix isolation spectroscopy and UV destruction of DME in an argon matrix was performed on a standard matrix isolation set-up (for a detailed description of the set-up, see Hudgins et al. 1994; Peeters et al. 2003) with a background pressure of $\sim 10^{-9}$ mbar and a CsI sample window thermally connected to a closed-cycle helium cryostat, capable of cooling down to $12 \mathrm{~K}$. The window and cryostat are rotatable without breaking the vacuum.

Argon (Praxair, 99.9990\%) and DME (Sigma-Aldrich, $99+\%)$ were lead through a cold trap cooled with a solid $\mathrm{CO}_{2}$ /acetone slush $\left(-80^{\circ} \mathrm{C}\right)$ to remove any trace of water and $\mathrm{CO}_{2}$ from the gas, before mixing them in a glass gas mixing set-up with a background pressure of $\sim 10^{-6}$ mbar and fitted with a capacitance manometer (Leybold). DME was diluted in argon to a ratio of 1:750.

The argon/DME mixture was deposited onto the $12 \mathrm{~K} \mathrm{CsI}$ substrate window to a thickness of $1 \mu \mathrm{m}$, controlled by standard laser interferometry. As a control, the DME column density was checked from the IR spectrum using the band strengths found by McKean et al. (1996), confirming the mixing ratio to $\sim 10 \%$ accuracy. A total layer thickness of $1 \mu \mathrm{m}$ allowed for full penetration of UV light through the sample.

UV irradiation was performed using a microwave-excited hydrogen-flow lamp (Opthos) with a flux of $4.8 \times 10^{14}$ photons $\mathrm{cm}^{-2} \mathrm{~s}^{-1}$. The lamp was calibrated using the $\mathrm{O}_{2}$ to $\mathrm{O}_{3}$ conversion method (actinometry) described by Cottin et al. (2003). UV irradiation was performed in $10 \mathrm{~s}$ intervals to a total of 2 min of irradiation, followed by 1 min intervals of irradiation until the DME sample was completely destroyed. After every UV irradiation interval, in situ IR spectra in the range 4000$500 \mathrm{~cm}^{-1}$ were recorded on an Excalibur FTS-4000 Fouriertransform IR spectrometer (Bio-Rad) at $1 \mathrm{~cm}^{-1}$ resolution, see Peeters et al. (2003).

\section{Results}

\subsection{UV photostability of DME}

IR spectra of DME condensed at low temperatures as well as the spectra of DME in $\mathrm{H}_{2} \mathrm{O}$, in an argon matrix and in a nitrogen matrix, followed by UV photolysis, have been reported by Schriver-Mazzuoli et al. (2002) and Schriver et al. (2004). We have measured the UV photodestruction rate of DME isolated in an argon matrix at $12 \mathrm{~K}$. In Fig. 1, IR spectra of DME after deposition (before UV irradiation) and after $10 \mathrm{~s}, 1 \mathrm{~min}$ and 10 min of irradiation are shown. Relevant bands of DME are found between 2990-2820 $\mathrm{cm}^{-1}$ (many small bands) and at $1456 \mathrm{~cm}^{-1}$ (double peak, $v_{14}$ and $\left.v_{19}\right), 1173 \mathrm{~cm}^{-1}\left(v_{16}\right)$, $1098 \mathrm{~cm}^{-1}\left(v_{17}\right)$ and $926 \mathrm{~cm}^{-1}\left(v_{6}\right)$, see McKean et al. (1996).

Upon irradiation with UV new features arose in the spectrum. These new bands are listed in Table 1. The photoproducts were identified and compared to the list of photoproducts reported by Schriver et al. (2004). The new bands found at 3020

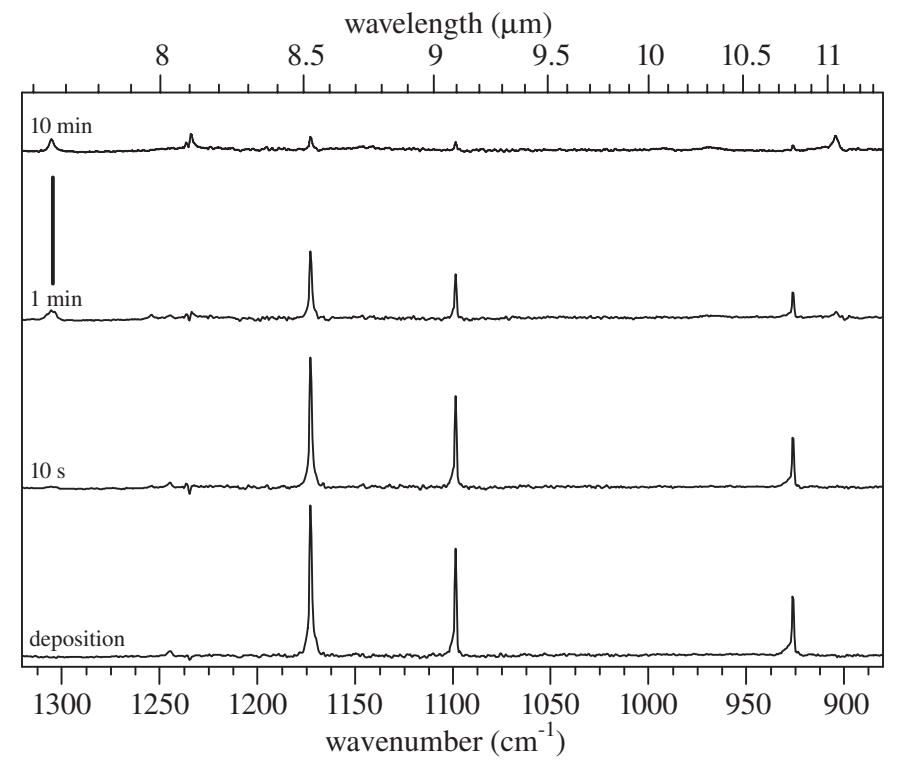

Fig. 1. The IR spectrum of DME isolated in argon at $12 \mathrm{~K}$ after deposition and after 10 seconds, 1 minute and 10 minutes of UV irradiation. The strongest vibrational transitions of DME can be found in the $1300-900 \mathrm{~cm}^{-1}$ region displayed here. The vertical bar scales to 0.01 absorbance units.

Table 1. Wavenumbers ( $\tilde{v})$ of the photoproducts that appeared after UV irradiation of matrix isolated DME. ${ }^{a}$ This work, ${ }^{b}$ Schriver et al. (2004), ${ }^{c}$ Jacox \& Olson (1987), ${ }^{d}$ not identified, ${ }^{e}$ Milligan \& Jacox (1973). The band found at $1863 \mathrm{~cm}^{-1}$, assigned to the HCO radical, was also found by Schriver et al. (2004), but only in a nitrogen matrix.

\begin{tabular}{ccl}
\hline \hline $\begin{array}{c}\tilde{v}^{a} \\
\left(\mathrm{~cm}^{-1}\right)\end{array}$ & $\begin{array}{c}\tilde{v}^{b} \\
\left(\mathrm{~cm}^{-1}\right)\end{array}$ & \\
\hline 3020 & 3024 & assignment $^{b}$ \\
& 2864 & $\mathrm{H}_{2} \mathrm{CO}$ \\
& 2797 & $\mathrm{H}_{2} \mathrm{CO}$ \\
2350 & 2345 & $\mathrm{CO}_{2}$ \\
2140 & 2138 & $\mathrm{CO}$ \\
2021 & & $\mathrm{HCCO}{ }^{c}$ \\
1863 & 1860 & $\mathrm{HCO}$ \\
& 1741 & $\mathrm{H}_{2} \mathrm{CO}$ \\
& 1498 & $\mathrm{H}_{2} \mathrm{CO}$ \\
1305 & 1305 & $\mathrm{CH}_{4}$ \\
1234 & & $-{ }^{d}$ \\
& 1039 & $\mathrm{O}_{3}$ \\
904 & & {$\left[\mathrm{HAr}_{2}\right]^{+e}$} \\
\hline
\end{tabular}

and $1305 \mathrm{~cm}^{-1}$ are attributed to methane, which is in agreement with the identification by Schriver et al. (2004). However, in contrast to Schriver et al. (2004) we did not find formaldehyde (bands at 2864, 2797, 1741 and $1498 \mathrm{~cm}^{-1}$ ), but instead a band appeared at $1863 \mathrm{~cm}^{-1}$, which was assigned to the radical HCO. Schriver et al. (2004) found this band only in the nitrogen matrix. Additionally, bands were detected at 2021 and $904 \mathrm{~cm}^{-1}$, which were tentatively assigned to HCCO (ethynyloxy radical, Jacox \& Olson 1987) and $\left[\mathrm{HAr}_{2}\right]^{+}($Milligan \& Jacox 1973), respectively. A third band found at $1234 \mathrm{~cm}^{-1}$ 
Table 2. Photon fluxes and DME destruction rates in the laboratory (lab), the diffuse interstellar medium (ISM), a dense cloud (DC), and the solar system at $1 \mathrm{AU}$ from the Sun (SoSy). $\phi_{\text {tot }}$ is the total flux of photons (photons $\mathrm{cm}^{-2} \mathrm{~s}^{-1}$ ) at wavelengths where DME destruction is efficient, and $k_{\mathrm{abs}}$ and $k_{\mathrm{destr}}$ are the destruction rates $\left(\mathrm{s}^{-1}\right)$ calculated respectively from gas phase absorption spectra and our experimental results. $\tau$ is the resulting half-life of DME in each environment.

\begin{tabular}{lcccl}
\hline \hline & $\begin{array}{c}\phi_{\text {tot }} \\
\left(\mathrm{cm}^{-2} \mathrm{~s}^{-1}\right)\end{array}$ & $\begin{array}{c}k_{\text {abs }} \\
\left(\mathrm{s}^{-1}\right)\end{array}$ & $\begin{array}{c}k_{\text {destr }} \\
\left(\mathrm{s}^{-1}\right)\end{array}$ & $\tau$ \\
\hline lab & $4.3 \times 10^{14}$ & $3.1 \times 10^{-3}$ & $1.3 \times 10^{-2}$ & $54 \mathrm{~s}$ \\
ISM & $1.4 \times 10^{8}$ & $1.3 \times 10^{-9}$ & $3.8 \times 10^{-9}$ & $5.8 \mathrm{yr}$ \\
DC & $1 \times 10^{3}$ & - & $2.7 \times 10^{-14}$ & $0.82 \mathrm{Myr}$ \\
SoSy & $6.7 \times 10^{12}$ & $2.9 \times 10^{-5}$ & $1.8 \times 10^{-4}$ & $64 \mathrm{~min}$ \\
\hline
\end{tabular}

could not be identified, but is probably due to contamination of the vacuum set-up.

All bands of DME diminished upon irradiation with UV light. The rate of destruction was calculated by plotting the natural log of the relative integrated peak area of the strongest bands at 1173, 1098 and $926 \mathrm{~cm}^{-1}$ (see Fig. 1) against irradiation time. The slope of a linear fit through these data points yielded the DME destruction rate, see Table 2.

\subsection{Spectroscopic properties of DME in ice matrices}

The IR spectrum of DME trapped in water ice has been reported by Schriver-Mazzuoli et al. (2002) for different temperatures in the range $11-160 \mathrm{~K}$. The authors showed that at low temperatures in a 1:10 mixed $\mathrm{DME} / \mathrm{H}_{2} \mathrm{O}$ ice the strongest bands can be found at 1161 and $1086 \mathrm{~cm}^{-1}$ (8.613 and $\left.9.208 \mu \mathrm{m}\right)$. No changes occur when the samples are heated to $110 \mathrm{~K}$. At $130 \mathrm{~K}$ these bands move to 1168 and $1096 \mathrm{~cm}^{-1}$ (8.562 and $9.124 \mu \mathrm{m}$ ), and their relative intensities change. Above $160 \mathrm{~K}$, DME disappears from the sample, because a change in crystal structure of the water ice allows trapped volatile species to escape.

Figure 2 shows a comparison between the laboratory IR spectrum of a 10:1 $\mathrm{H}_{2} \mathrm{O} / \mathrm{DME}$ mixture (top) and the ISO SWS spectrum of high mass protostar W33A (bottom). The DME fundamental transitions are identified by vertical bands. The DME bands in the $3000 \mathrm{~cm}^{-1}(3.3 \mu \mathrm{m})$ region have low intrinsic band strengths. Additionally, they are mixed into the wing of the $\mathrm{OH}$ bending mode of $\mathrm{H}_{2} \mathrm{O}$ that falls in the 3125$2778 \mathrm{~cm}^{-1}(3.2-3.6 \mu \mathrm{m})$ range.

The two DME peaks visible in the laboratory spectrum at $1461 \mathrm{~cm}^{-1}(6.84 \mu \mathrm{m})$ and $1455 \mathrm{~cm}^{-1}(6.87 \mu \mathrm{m})$ are obscured in the W33A spectrum by the band at $\sim 1460 \mathrm{~cm}^{-1}(6.85 \mu \mathrm{m})$, which was ascribed to the $v_{4}$ transition of $\mathrm{NH}_{4}^{+}$(Schutte \& Khanna 2003). The strongest peaks in the DME spectrum, $1173 \mathrm{~cm}^{-1}(8.53 \mu \mathrm{m}), 1098 \mathrm{~cm}^{-1}(9.11 \mu \mathrm{m})$ and $926 \mathrm{~cm}^{-1}$ $(10.8 \mu \mathrm{m})$, see Sect. 3.1 , are completely obscured by the strong silicate bands at $1000 \mathrm{~cm}^{-1}(10 \mu \mathrm{m})$. A comparison between the laboratory $\mathrm{DME} / \mathrm{H}_{2} \mathrm{O}$ IR spectrum and the ISO SWS spectrum of W33A predicts that it will be difficult to detect DME in the solid state. The DME bands are likely to be obscured by other stronger bands, from more abundant species. Furthermore,

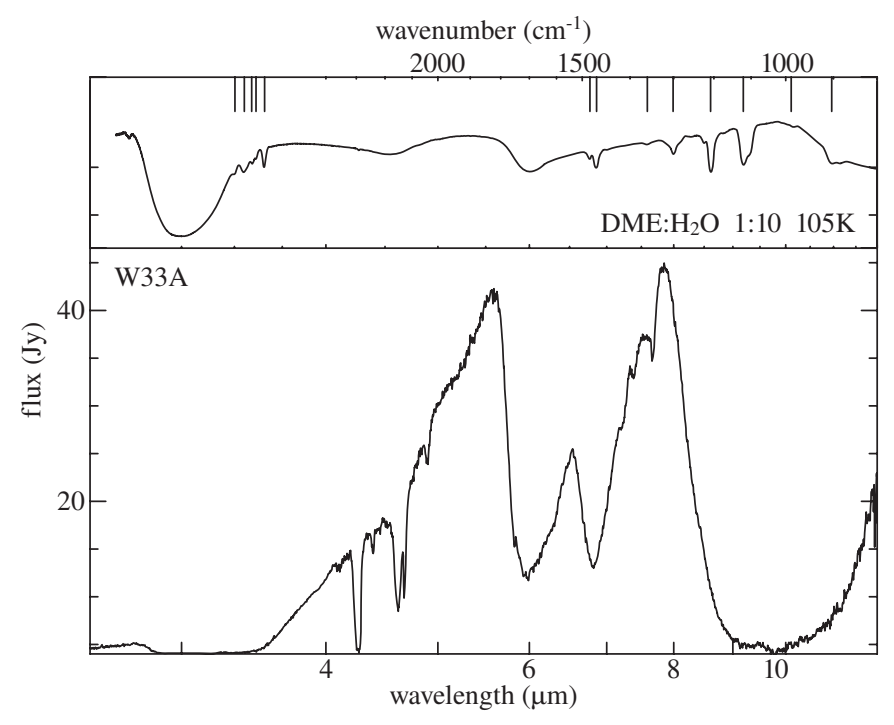

Fig. 2. Comparison of a 1:10 DME/ $\mathrm{H}_{2} \mathrm{O}$ mixture at $105 \mathrm{~K}$ (top) with the ISO SWS spectrum of the high mass embedded protostar W33A (bottom). The vertical lines identify the DME bands.

the laboratory spectrum was made with a $\mathrm{DME} / \mathrm{H}_{2} \mathrm{O}$ ratio of $10 \%$, while a much lower $\mathrm{DME} / \mathrm{H}_{2} \mathrm{O}$ ratio is expected to be present in $\mathrm{H}_{2} \mathrm{O}$ dominated ices $(\sim 0.02-0.2 \%)$.

\section{Discussion}

\subsection{DME photodestruction in different astronomical environments}

Photoabsorption cross sections for gas phase DME have been measured by numerous groups (see for example Fig. 5 of Feng et al. 2000). These experiments showed that the cross section varies strongly with wavelength, peaking at $\sim 80 \mathrm{~nm}$ (Koizumi et al. 1986). Crovisier (1994) reported a photodestruction rate in the solar system at $1 \mathrm{AU}$ from a quiet sun of $3.11 \times 10^{-5} \mathrm{~s}^{-1}$ (with a contribution of $6.6 \times 10^{-6} \mathrm{~s}^{-1}$ due to Ly- $\alpha$ ), based on the absorption data by Suto et al. (1988).

In the experiments described in Sect. 2 we have measured the destruction rate of DME directly, upon irradiation with a hydrogen lamp. The spectrum of this lamp, as measured by Cottin et al. (2003) is shown in Fig. 3 (dashed line). The spectrum of Cottin et al. (2003) was scaled to yield an integrated flux of $4.8 \times 10^{14}$ photons $\mathrm{cm}^{-2} \mathrm{~s}^{-1}$, the value obtained by calibration of our lamp, also described by Cottin et al. (2003). The DME absorption spectrum as measured by Suto et al. (1988) is also shown in Fig. 3 (solid line). The strongest emission line in the lamp spectrum, around 156-168 nm, overlaps with the $\tilde{D}$ transition of DME, which results in a high absorption rate. The absorption cross section of DME is much higher at $121 \mathrm{~nm}$, but the Ly- $\alpha$ emission of the hydrogen lamp contains at most 5\% of the total energy between 100-200 nm (Cottin et al. 2003).

Also shown in Fig. 3 are the spectrum of a quiet sun at 1 AU (dash-dotted line, ASTM E490-AM0 spectrum, see http://rredc.nrel.gov/solar/spectra/amQ/) and the Draine field between 100-200 nm (dotted line, Draine 1978; 


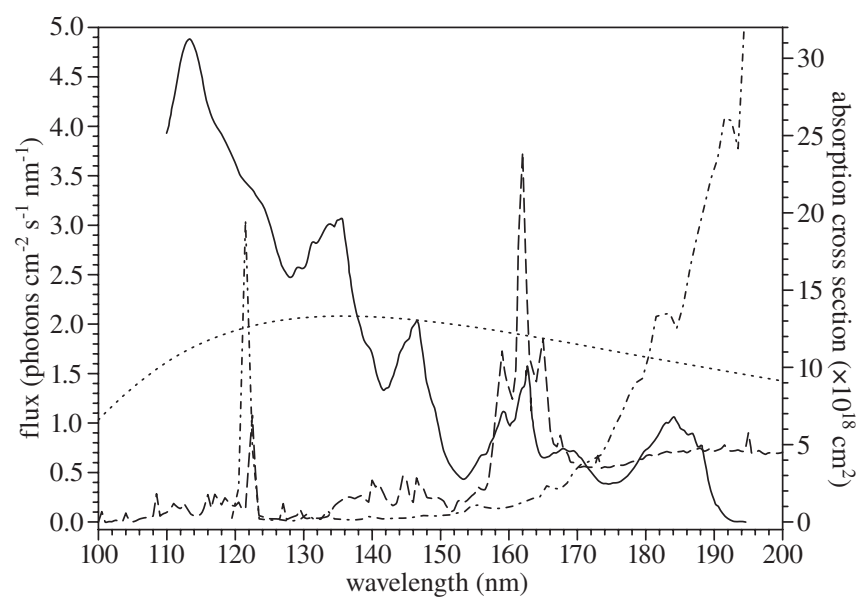

Fig. 3. A comparison of different astronomical UV sources with the DME absorption spectrum (solid line) in the range 100-200 nm. The sources shown here are the laboratory hydrogen lamp (dashed line, Cottin et al. 2003), the ASTM E490-AM0 solar spectrum (dashdotted line), and the Draine interstellar field (dotted line, Draine 1978; Roberge et al. 1991). The UV sources scale to the left $Y$-axis by a factor $10^{13}$ for the hydrogen lamp, $10^{11}$ for the solar spectrum and $10^{6}$ for the Draine field. The DME absorption spectrum scales to the right $Y$-axis.

Roberge et al. 1991). The solar spectrum has a larger Ly- $\alpha$ contribution to the total energy delivered between 100-200 nm ( $\sim 9 \%)$ compared to the hydrogen lamp.

The spectra in Fig. 3 can be used to calculate the UV absorption rate of DME for the three different UV fields. The interval in which all four traces overlap lies between 119 and $195 \mathrm{~nm}$. We used these wavelengths as the integration limits for the following calculations. The UV absorption rate $k_{\mathrm{abs}}\left[\mathrm{s}^{-1}\right]$ is given by:

$k_{\mathrm{abs}}=\int_{119}^{195} \sigma_{\mathrm{abs}}(\lambda) \phi(\lambda) \mathrm{d} \lambda$

where $\sigma_{\text {abs }}(\lambda)$ is the absorption cross section $\left[\mathrm{cm}^{2}\right]$ and $\phi(\lambda)$ is the UV flux (photons $\mathrm{cm}^{-2} \mathrm{~s}^{-1} \mathrm{~nm}^{-1}$ ) at wavelength $\lambda[\mathrm{nm}]$. When the destruction rates are calculated in this way, we find values of $1.3 \times 10^{-9} \mathrm{~s}^{-1}$ for the Draine field in the diffuse interstellar medium and $2.9 \times 10^{-5} \mathrm{~s}^{-1}$ for the solar system, see Table 2 . These numbers are comparable to those given by Roberge et al. (1991) and Crovisier (1994), respectively, although slightly lower. This can be explained by the fact that the integration here is done over the wavelength range 119$195 \mathrm{~nm}$, while others have used the full range of data available. The calculation was also done for the hydrogen lamp and yielded a rate of $3.1 \times 10^{-3} \mathrm{~s}^{-1}$.

Our experimental data yield a destruction rate of $1.3 \times$ $10^{-2} \mathrm{~s}^{-1}$, which is larger than the expected theoretical value of $3.1 \times 10^{-3} \mathrm{~s}^{-1}$. In order to extrapolate our results to the photodestruction rate of DME in space, we calculated the mean absorption cross section, $\bar{\sigma}$, of DME for photons with energies between 119-195 nm. From the DME absorption spectrum shown in Fig. 3 we derive $\bar{\sigma}=2.7 \times 10^{-17} \mathrm{~cm}^{2}$. The destruction rate of DME in different environments can then be approximated by

$k_{\mathrm{destr}}=\bar{\sigma} \phi_{\mathrm{tot}}$ where $\phi_{\text {tot }}$ is the integrated UV flux between 119 and $195 \mathrm{~nm}$. Table 2 lists the derived destruction rates, as well as the rates calculated via integration of the absorption spectrum $\left(k_{\mathrm{abs}}\right.$, Eq. (3)).

An important difference between $k_{\text {abs }}$ and $k_{\text {destr }}$ is that the former was measured in hot $(\sim 290 \mathrm{~K})$ gas phase, while the latter was measured in a cold $(12 \mathrm{~K})$ argon matrix. At first glance, the destruction in the argon matrix would be expected to be slower than the gas phase, because the energy of the UV photons can be dissipated into the matrix. Also, because the reaction products are confined to a small space in the argon matrix, back-formation of the original molecules could occur. However, we find that the destruction in the matrix actually goes faster in the argon matrix. Contaminants in the matrix could enhance the destruction reaction, but we dismiss the idea of contaminants, because the most abundant and reactive contaminant, $\mathrm{H}_{2} \mathrm{O}$, is present in such low concentration, that it falls below the IR detection limit. This means that if water would be present in the matrix, the $\mathrm{H}_{2} \mathrm{O} / \mathrm{DME}$ ratio would be $<0.02$.

The destruction rates are easily converted into half-lives, the time in which $50 \%$ of an initial amount of DME is destroyed. The half-life $\tau$ [s] is equal to $\ln 2 / k_{\text {destr }}$. In Table 2 the half-life is also calculated for a dense cloud environment. In a dense cloud the UV flux is $1 \times 10^{3}$ photons $\mathrm{cm}^{-2} \mathrm{~s}^{-1}$, according to Prasad \& Tarafdar (1983). The UV field in this environment is believed to be comparable to the hydrogen lamp, albeit with a lower total flux, since both UV fields are generated by hydrogen emission. This means that the destruction rate in dark clouds can be found by scaling the destruction rate in the lab with the ratio of the integrated fluxes: $10^{3} / 4.8 \times 10^{14}$. The resulting half-life of $8.2 \times 10^{5}$ year means that DME could survive the average lifetime $\left(\sim 10^{6}\right.$ year $)$ of a dense cloud (Elmegreen 2000; Hartmann et al. 2001).

\subsection{DME in hot molecular cores}

In order to investigate whether DME is formed solely by reaction 1 or if a grain reaction is required, we have performed model calculations of hot core chemistry. The high visual extinction of hot cores $(\sim 300 \mathrm{mag})$ means that photodestruction is only possible through the Prasad-Tarafdar radiation field. Thus, we also considered the effect of these photons in the postevaporation chemistry.

We used our standard hot core model as described in Rodgers \& Charnley (2001a), and assumed a temperature of $100 \mathrm{~K}$, a density of $n\left(\mathrm{H}_{2}\right)=10^{7} \mathrm{~cm}^{-3}$, and a cosmic ray ionization rate, $\zeta$, of $1.3 \times 10^{-17} \mathrm{~s}^{-1}$. Our only alterations to the reaction scheme were to turn off three radiative association reactions involving $\mathrm{CH}_{3}^{+}$: those with water, methanol, and acetaldehyde which form $\mathrm{CH}_{3} \mathrm{OH}_{2}^{+},\left(\mathrm{CH}_{3}\right)_{2} \mathrm{OH}^{+}$, and $\mathrm{C}_{3} \mathrm{H}_{7} \mathrm{O}^{+}$, respectively. Initial abundances are as in Rodgers \& Charnley (2001a), except for water which we injected with $n\left(\mathrm{H}_{2} \mathrm{O}\right) / n\left(\mathrm{H}_{2}\right)=6 \times 10^{-5}$. The initial ammonia abundance was set to zero. In the Rodgers \& Charnley model, DME is formed in the gas phase almost entirely via reaction 1 , with a very small contribution due to the radiative association of $\mathrm{CH}_{3}^{+}$and methanol. DME is destroyed by protonation (it is assumed $50 \%$ 
of the dissociative recombinations of $\left(\mathrm{CH}_{3}\right)_{2} \mathrm{OH}^{+}$lead back to $\mathrm{DME}+\mathrm{H}$, and $50 \%$ result in the fragmentation to methanol and $\mathrm{CH}_{3}$ ), and by reactions with $\mathrm{He}^{+}, \mathrm{H}^{+}, \mathrm{C}^{+}$, and $\mathrm{CH}_{3}^{+}$. In model runs with the Prasad-Tarafdar radiation field included, DME was also destroyed by photons, with a rate of $2833 \times \zeta$.

We computed models for three specific scenarios.

(a) A reference hot core model similar to that of Rodgers \& Charnley (2001a), as described above, both with and without the effects of the Prasad-Tarafdar radiation field. Ices were evaporated instantaneously and the subsequent chemistry followed.

(b) As in the models of (a), except that reaction 1 was turned off and DME was instead injected from grains at an abundance of $n\left(\mathrm{CH}_{3} \mathrm{OCH}_{3}\right) / n\left(\mathrm{H}_{2}\right)=1 \times 10^{-7}$, consistent with observed upper limits in interstellar ices (see Sect. 3.2).

(c) As in the models of (b), except that the injected methanol abundance was lowered to equal that of DME.

Figure 4 shows the chemical evolution in each scenario. From Fig. 4a it can be seen that Prasad-Tarafdar photons have little effect on the methanol-DME chemistry. This is because, due to the relatively large proton affinities of DME and methanol, the dominant ions in these models are $\left(\mathrm{CH}_{3}\right)_{2} \mathrm{OH}^{+}$and $\mathrm{CH}_{3} \mathrm{OH}_{2}^{+}$. The major loss route for methanol is in fact the self-methylation in reaction 1 , whereas the protonation of DME, eventually formed from the recombination of $\left(\mathrm{CH}_{3}\right)_{2} \mathrm{OH}^{+}$, is mainly by $\mathrm{CH}_{3} \mathrm{OH}_{2}^{+}$. Hence, $\mathrm{CH}_{3} \mathrm{OH}_{2}^{+}$dominates both the production and loss of DME in hot cores. In the cases where reaction 1 was assumed not to occur, Figs. $4 \mathrm{~b}$ and c show that DME injection from dust leads to a different chemical evolution.

In these models the DME is again destroyed through protonation and the methanol abundance actually increases due to the fact that it is a product of one of the dissociative electron recombination channels of $\left(\mathrm{CH}_{3}\right)_{2} \mathrm{OH}^{+}$. In cases (b) and (c), Prasad-Tarafdar photons can affect the long-term destruction of methanol, however, even increasing the cosmic ray ionization rate to $5 \times 10^{-17} \mathrm{~s}^{-1}$ (not shown) only produces marginally faster destruction of DME.

Injection of DME from dust leads to its peak abundance occurring much earlier in the evolution, and so would suggest much younger ages, of typically less than $10^{4}$ years, than when it forms solely by gas phase chemistry (i.e. $\gtrsim 10^{4}$ years, see Fig. 4a). Only a model with an initially low methanol abundance can reproduce and maintain $\mathrm{CH}_{3} \mathrm{OCH}_{3} / \mathrm{CH}_{3} \mathrm{OH} \sim 1$. Based on this ratio the maximum core ages would be less than a few thousand years. In a hot core rich in methanol, it is clear from Figs. 4 that DME production through reaction 1 can dominate any grain-surface contribution, unless they happen to be observed very early in their evolution.

Thus, one might ask if, in general, interstellar DME could come from both sources, where the grain-surface contribution only becomes evident in the presence of relatively methanolpoor ices? Unlike many proposed pathways to the formation of other complex interstellar molecules (e.g. Charnley 2001), reaction 1 is experimentally verified and, as shown in Fig. 4a, can reproduce the observed DME abundances. Only three studies, based on single-dish observations, have thus far presented derived ratios of $\mathrm{CH}_{3} \mathrm{OCH}_{3} / \mathrm{CH}_{3} \mathrm{OH} \sim 1$
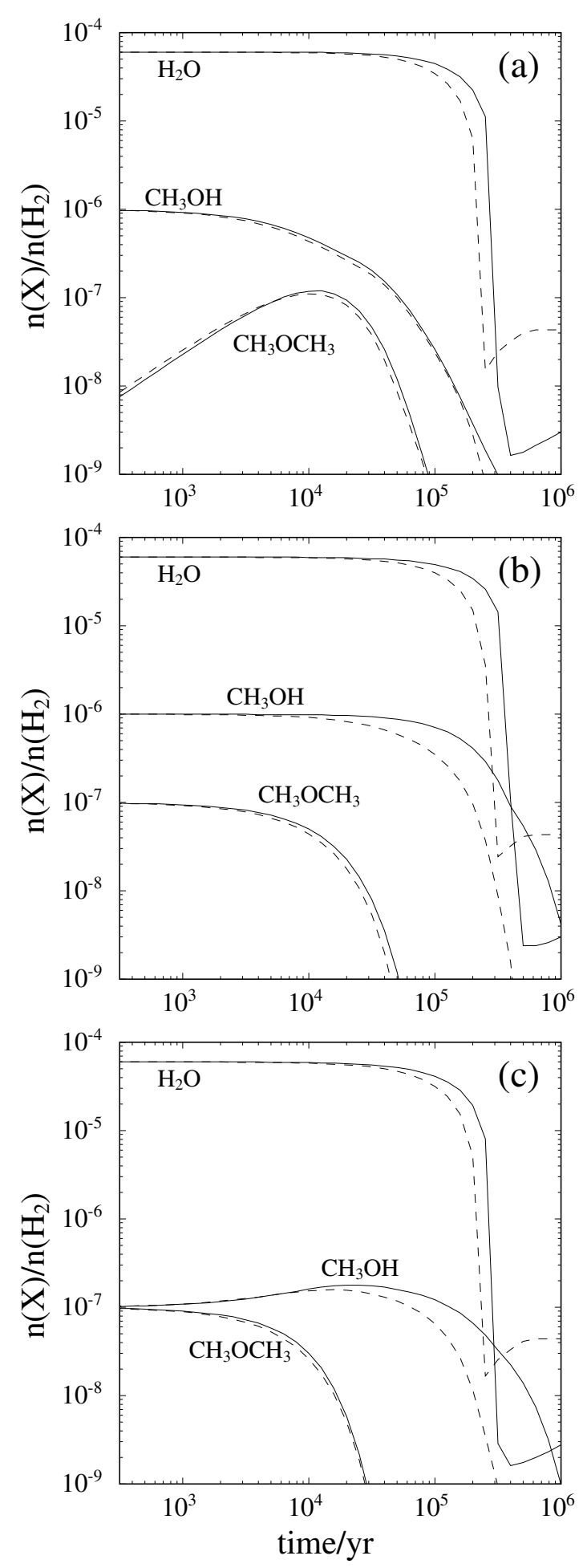

Fig. 4. Hot core evolution of water, methanol and DME in three model scenarios. Broken curves are models which include photodestruction from the Prasad-Tarafdar radiation field. See text for details.

(MacDonald et al. 1996; Helmich \& van Dishoeck 1997; Cazaux et al. 2003). Cazaux et al. (2003) determined that, when combined with data from Schöier et al. (2002), the abundance ratios of many O-containing molecules in IRAS 162932422 relative to methanol were almost all much higher than found elsewhere. In particular, Cazaux et al. (2003) argued that an observed ratio of $\mathrm{HCOOCH}_{3} / \mathrm{CH}_{3} \mathrm{OH} \sim 1$ would 
rule out gas phase production, as would a similarly high ratio involving DME. Although grain-surface production of $\mathrm{HCOOCH}_{3}$ is quite possible (see Sect. 1.1), a serious problem for this interpretation of the origin of DME lies in the fact that Cazaux et al. (2003) formed their $\mathrm{HCOOCH}_{3} / \mathrm{CH}_{3} \mathrm{OH}$ and $\mathrm{CH}_{3} \mathrm{OCH}_{3} / \mathrm{CH}_{3} \mathrm{OH}$ ratios using abundances from two different set of observations. Schöier et al. (2002) derived their methanol abundances using a gas column density of $N\left(\mathrm{H}_{2}\right)=1.6 \times 10^{24} \mathrm{~cm}^{-2}$, whereas Cazaux et al. (2003) used $N\left(\mathrm{H}_{2}\right)=7.5 \times 10^{22} \mathrm{~cm}^{-2}$ in deriving their $\mathrm{HCOOCH}_{3}$ and $\mathrm{CH}_{3} \mathrm{OCH}_{3}$ abundances. Interferometric observations show that IRAS 16293-2422 can be resolved into two so-called "hot corinos" (Kuan et al. 2004; Bottinelli et al. 2004; Huang et al. 2005). In this case, abundances derived using more realistic (i.e. larger) values of $N\left(\mathrm{H}_{2}\right) \gtrsim 10^{24} \mathrm{~cm}^{-2}$ lead to $\mathrm{HCOOCH}_{3}$ and $\mathrm{CH}_{3} \mathrm{OCH}_{3}$ abundances $\left(\sim 10^{-9}-10^{-8}\right)$, and ratios relative to $\mathrm{CH}_{3} \mathrm{OH} \sim 0.01-0.1$, that are more in accord with those predicted by theory (Kuan et al. 2004; Bottinelli et al. 2004; Huang et al. 2005).

\section{Conclusions}

We have considered the chemistry of DME in astronomical environments from experimental, observational and theoretical perspectives. We specifically addressed issues relating to the survivability of DME against UV destruction, its actual formation mechanism, and its chemistry around massive protostars.

- We investigated the UV photostability of DME in the laboratory. We compared our results for UV photodestruction of DME in argon matrices with previous work for DME in water ice matrices and in the gas phase. All bands of DME diminished upon irradiation with UV light. The major photoproducts which could be identified include methane, HCO and $\mathrm{HCCO}$.

- We used our laboratory measurements to estimate the lifetime of gaseous DME under various astronomical conditions. The half-life of DME in the diffuse interstellar medium corresponds to 5.8 years, in a dense cloud it corresponds to $0.8 \mathrm{Myr}$ and in the solar system at $1 \mathrm{AU}$ distance DME can only survive for $64 \mathrm{~min}$. The low photostability of DME could be a reason why DME has not been detected in the cometary coma.

- We compared laboratory IR spectra of DME/water ice mixtures with the IR spectra of the astronomical ices surrounding a forming massive protostar W33A. We find that the strongest bands of DME are obscured by the dominant bands of water ice and silicates in interstellar spectra. The detection of interstellar solid DME is therefore a very difficult task.

- We have modelled the gas phase chemistry of DME in hot molecular cores. We find that inclusion of Prasad-Tarafdar photons has a negligible effect on its chemical evolution. The high efficiency of DME production by gas phase chemistry, coupled with its observed quite low upper limits in ices, suggest that grain-surface reactions are at most a minor source of interstellar DME.
Given the difficulty in detecting DME in interstellar ices, searches for deuterated isotopomers of DME in hot cores and setting more stringent upper limits on the abundance of DME in comets (cf. Crovisier et al. 2004) would appear to be most helpful for assessing the potential importance of a grain-surface contribution. However, applying Ockham's Razor leads us to the conclusion that gas phase synthesis via the self-methylation of methanol is the chemical pathway to DME in space.

Acknowledgements. P.E. and Z.P. are supported by grant NWOVI 016.023.003. S.B.C. and S.D.R. were supported by NASA's Exobiology and LTSA Programs through funds allocated by NASA Ames under Cooperative Agreement No. NCC2-1412 to the SETI Institute.

\section{References}

Allamandola, L., Bernstein, M., \& Sandford, S. 1997, Astronomical $\&$ biochemical origins and the search for life in the universe, ed. C. Cosmovici, S. Bowyer, \& D. Wertheimer (Bologna: Editrice Compositori), 23

Allen, M., \& Robinson, G. 1977, ApJ, 212, 396

Bernstein, M., Sandford, S., Allamandola, L., Chang, S., \& Scharberg, M. 1995, ApJ, 454, 327

Blake, G., Sutton, E., Masson, C., \& Phillips, T. 1987, ApJ, 315, 621

Bockelée-Morvan, D., Crovisier, J., Mumma, M., \& Weaver, H. 2005, in COMETS II, ed. M. Festou, H. Keller, \& H. Weaver (Tuscon: University of Arizona)

Bottinelli, S., Ceccarelli, C., Lefloch, B., et al. 2004, ApJ, 615, 354

Caselli, P., Hasegawa, T., \& Herbst, E. 1993, ApJ, 408, 548

Cazaux, S., Tielens, A., Ceccarelli, C., et al. 2003, ApJ, 593, 51

Charnley, S. 1997a, Astronomical \& biochemical origins and the search for life in the universe, ed. C. Cosmovici, S. Bowyer, \& D. Wertheimer (Bologna: Editrice Compositori), 89

Charnley, S. 1997b, ApJ, 481, 396

Charnley, S. 2001, The bridge between the big bang and biology, ed. F. Giovanelli (Rome: Consiglio Nazionale delle Ricerche), 139 Charnley, S., Tielens, A., \& Millar, T. 1992, ApJ, 399, L71

Charnley, S., Kress, M., Tielens, A., \& Millar, T. 1995, ApJ, 448, 232

Cottin, H., Moore, M., \& Bénilan, Y. 2003, ApJ, 590, 874

Crovisier, J. 1994, JGR E, 99, 3777

Crovisier, J., Bockelée-Morvan, D., Colom, P., et al. 2004, A\&A, 418, 1141

Draine, B. 1978, ApJS, 36, 595

Ehrenfreund, P., \& Charnley, S. 2000, ARA\&A, 38, 427

Ehrenfreund, P., Bernstein, M., Dworkin, J., Sandford, S., \& Allamandola, L. 2001a, ApJ, 550, L95

Ehrenfreund, P., d'Hendecourt, L., Charnley, S., \& Ruiterkamp, R. 2001b, JGR E, 106, 33291

Elmegreen, B. 2000, ApJ, 530, 277

Feng, F., Cooper, G., \& Brion, C. 2000, Chem. Phys., 260, 391

Friberg, P., Madden, S., Hjalmarson, A., \& Irvine, W. 1988, A\&A, 195,281

Gerakines, P., Moore, M., \& Hudson, R. 2001, JGR E, 106, 33381

Hartmann, L., Ballesteros-Paredes, J., \& Bergin, E. 2001, ApJ, 562, 852

Hatchell, J., Thompson, M., Millar, T., \& MacDonald, G. 1998, A\&A, 338,713

Helmich, F., \& van Dishoeck, E. 1997, A\&AS, 124, 205

Hollis, J., \& Churchwell, E. 2001, ApJ, 551, 803

Horn, A., Møllendal, H., Sekiguchi, O., et al. 2004, ApJ, 611, 605

Huang, H.-C., Kuan, Y.-J., Charnley, S., Hirano, H., \& Takakuwa, S. 2005, Adv. Space Rev., submitted 
Hudgins, D., Sandford, S., \& Allamandola, L. 1994, J. Phys. Chem., 98,4243

Ikeda, M., Ohishi, M., Nummelin, A., et al. 2001, ApJ, 560, 792

Jacox, M., \& Olson, W. 1987, J. Chem. Phys, 86, 3134

Karpas, Z., \& Mautner, M. 1989, J. Phys. Chem, 93, 1859

Koizumi, H., Hironaka, K., Shinsaka, K., et al. 1986, J. Chem. Phys, 85,4276

Kuan, Y., Huang, H., Charnley, S., et al. 2004, ApJ, 616, L27

Kurtz, S., Cesaroni, R., Churchwell, E., Hofner, P., \& Walmsley, C. 2000, Protostars and Planets IV, ed. V. Mannings, A. Boss, \& S. Russell (Tucson: University of Arizona Press), 229

Le Teuff, Y., Millar, T., \& Markwick, A. 2000, A\&AS, 146, 157

MacDonald, G., Gibb, A., Habing, R., \& Millar, T. 1996, A\&AS, 119, 333

McKean, D., Kindness, A., Wilkie, N., \& Murphy, W. 1996, Spectrochim. Acta A, 52, 445

Mehringer, D., \& Snyder, L. 1996, ApJ, 471, 897

Milligan, D., \& Jacox, M. 1973, J. Mol. Spectrosc, 46, 460

Nummelin, A., Bergman, P., Hjalmarson, A., et al. 2000, ApJS, 128, S213

Peeters, Z., Botta, O., Charnley, S., Ruiterkamp, R., \& Ehrenfreund, P. 2003, ApJ, 593, L129
Peeters, Z., Botta, O., Charnley, S., et al. 2005, A\&A, 433, 583

Prasad, S., \& Tarafdar, S. 1983, ApJ, 267, 603

Roberge, W., Jones, D., Lepp, S., \& Dalgarno, A. 1991, ApJS, 77, S287

Rodgers, S., \& Charnley, S. 2001a, ApJ, 546, 324

Rodgers, S., \& Charnley, S. 2001b, MNRAS, 323, 84

Schöier, F., Jørgensen, J., van Dishoeck, E., \& Blake, G. 2002, A\&A, 390,1001

Schriver, A., Coanga, J., Schriver-Mazzuoli, L., \& Ehrenfreund, P. 2004, Chem. Phys. Lett., 386, 377

Schriver-Mazzuoli, L., Coanga, J., Schriver, A., \& Ehrenfreund, P. 2002, Vib. Spectrosc., 30, 245

Schutte, W., \& Khanna, R. 2003, A\&A, 398, 1049

Schutte, W., Tielens, A., Whittet, D., et al. 1996, A\&A, 315, 333

Sorrell, W. 2001, ApJ, 555, L129

Stäuber, P., Doty, S., van Dishoeck, E., Jørgensen, J., \& Benz, A. 2004, A\&A, 425, 577

Suto, M., Chao, Y., \& Lee, L. 1988, J. Chem. Phys, 89, 6555

Sutton, E., Peng, R., Danchi, W., et al. 1995, ApJS, 97, 455

Turner, B. 1991, ApJS, 76, 617

van Dishoeck, E., \& Blake, G. 1998, ARA\&A, 36, 317

Watanabe, N., \& Kouchi, A. 2002, ApJ, 571, 173 